\title{
Collisional Granular Flow as a Micropolar Fluid
}

\author{
Namiko Mitarai ${ }^{1}$, Hisao Hayakawa ${ }^{2}$, and Hiizu Nakanishi ${ }^{1}$ \\ ${ }^{1}$ Department of Physics, Kyushu University 33, Fukuoka, 812-8581, Japan \\ ${ }^{2}$ Graduate School of Human and Environmental Studies, \\ Kyoto University, Sakyo-ku, Kyoto 606-8501, Japan
}

(Dated: November 1, 2018)

\begin{abstract}
We show that a micropolar fluid model successfully describes collisional granular flows on a slope. A micropolar fluid is the fluid with internal structures in which coupling between the spin of each particle and the macroscopic velocity field is taken into account. It is a hydrodynamical framework suitable for granular systems which consists of particles with macroscopic size. We demonstrate that the model equations can quantitatively reproduce the velocity and the angular velocity profiles obtained from the numerical simulation of the collisional granular flow on a slope using a simple estimate for the parameters in the theory.
\end{abstract}

In spite of the long history of research on the granular flows, the theoretical framework for their rheology has not yet been established. One factor that makes analytical treatment difficult is that there is not a great separation of the length scales; the size of each particle is often comparable with the scale of the macroscopic collective motion. Therefore, there are many situations in which simple hydrodynamic approaches cannot be used to characterize granular flows [1]. Even when we consider the rapid granular flows [2], in which the density is low enough that kinetic theory seems to be valid, the coupling between the rotation of each particle and macroscopic velocity field may not be negligible. Thus, the behavior of the flow can deviate from that of a Newtonian fluid.

The micropolar fluid model is a continuum model to describe a fluid that consists of particles with spinning motion [3]. The model equations include an asymmetric stress tensor and a couple stress tensor. Therefore, the model can be a suitable framework to describe granular flows.

Although some research on the application of the micropolar fluid model to granular flows has been carried out [4], most work has considered dense granular flows, in which the constitutive equations adopted for the stress and the couple stress tensors were very complicated. Hence it was difficult to interpret the results physically.

In this paper, we apply a micropolar fluid model to a collisional granular flow. We adopt a set of constitutive equations that are a simple and natural extension of those for a Newtonian fluid. Note that Newtonian fluid models of rapid granular flows [2] have been well established. We calculate the velocity and angular velocity profiles for uniform, steady flow on a slope and demonstrate that the micropolar fluid model reproduces the results of numerical simulations.

It is easy to derive the equations for the number density $n$, the velocity $v_{i}$, and the microrotation field $\omega_{i}$ of a system that consists of identical particles with mass $m$ and moment of inertia $I$. From the conservation laws of mass, momentum, and angular momentum [3], we obtain

$$
D_{t} n=-n \partial_{k} v_{k}
$$

$$
\begin{aligned}
m n D_{t} v_{i} & =m n f_{i}+\partial_{j} S_{j i}, \\
I_{n} D_{t} \omega_{i} & =\partial_{j} C_{j i}+s_{i}^{(a)} .
\end{aligned}
$$

The summation convention applies to repeated subscripts. $\partial_{i}$ represents a partial derivative with respect to the $i$ th coordinate, $D_{t} \equiv \partial / \partial t+v_{k} \partial_{k}$ is Lagrange's derivative, and $f_{i}$ is the body force per unit mass. Here, $S_{i j}$ and $C_{i j}$ are the stress tensor and the couple stress tensor that, respectively, represents the $j$ component of the surface force and the surface torque acting on the plane perpendicular to the $i$ axis per unit area, and $s_{i}^{(a)}$ is the torque due to the asymmetric part of the stress tensor defined as

$$
s_{i}^{(a)}=\epsilon_{i j k} S_{j k},
$$

where $\epsilon_{i j k}$ is the alternating tensor of Levi-Civita.

For the constitutive equation of the stress tensor $S_{i j}$, we adopt [3]:

$$
\begin{aligned}
S_{i j}= & \left(-p+\lambda \partial_{k} v_{k}\right) \delta_{i j}+\mu\left(\partial_{i} v_{j}+\partial_{j} v_{i}\right) \\
& +\mu_{r}\left[\left(\partial_{i} v_{j}-\partial_{j} v_{i}\right)-2 \epsilon_{i j k} \omega_{k}\right],
\end{aligned}
$$

with $\delta_{i j}$, Kronecker's delta. The symmetric part of $S_{i j}$ in Eq. (5) is the same as the stress tensor of the Newtonian fluid with pressure $p$, shear viscosity $\mu$, and bulk viscosity $\lambda$. The third term on the right hand of Eq. (5) represents the asymmetric part of the stress tensor due to the difference between the rotation of the mean velocity field and particles' own spin. This gives $\boldsymbol{s}^{(a)}=2 \mu_{r}[\nabla \times \boldsymbol{v}-2 \boldsymbol{\omega}]$, using Eq. (四). The microrotation viscosity $\mu_{r}$ represents the coupling between the velocity and the microrotation field. For the couple stress tensor $C_{i j}$, we use the theorem that isotropic second order tensors can be expanded as the trace, the symmetric part, and the asymmetric part of rate of strain tensor for microrotation [3]:

$$
\begin{aligned}
C_{i j}= & c_{0} \partial_{k} \omega_{k} \delta_{i j}+\frac{\mu_{B}+\mu_{A}}{2}\left(\partial_{i} \omega_{j}+\partial_{j} \omega_{i}\right) \\
& +\frac{\mu_{B}-\mu_{A}}{2}\left(\partial_{i} \omega_{j}-\partial_{j} \omega_{i}\right),
\end{aligned}
$$

where the coefficients of angular viscosity are $c_{0}, \mu_{A}$, and $\mu_{B}$. It should be noted that the dimensions of these 
coefficients are different from the viscosities in the stress tensor by length-squared because of the difference of the dimensions between $S_{i j}$ and $C_{i j}$ and between $v_{i}$ and $\omega_{i}$.

It is debatable whether such a straightforward extension of Newtonian constitution relations can be applied to granular flow, because the hydrostatic term in a granular material should have a different form. For a collisional flow, however, we expect that such an effect is not important. Therefore, we concentrate on a collisional granular flow.

The coefficients of viscosity that appear in Eqs. (5) and (16) have been derived based on the kinetic theory of three-dimensional spheres with rough surfaces 5 , 6 . Here, for later convenience, and also to make the physical meaning of the model clear, we briefly summarize the rough estimate of the coefficients of viscosity to the lowest order for two-dimensional disks using elementary kinetic theory. Let us consider a two-dimensional fluid that consists of identical disks with diameter $\sigma$ and that is flowing uniformly in the $x$ direction, namely $n=n(y)$, $\boldsymbol{v}=(u(y), 0,0)$, and $\boldsymbol{\omega}=(0,0, \omega(y))$. Then we have

$$
S_{y x}=\mu u^{\prime}(y)+\mu_{r}\left[u^{\prime}(y)+2 \omega(y)\right]
$$

and

$$
C_{y z}=\mu_{B} \omega^{\prime}(y)
$$

where the prime denotes a derivative with respect to its argument. Here, $S_{y x}\left(C_{y z}\right)$ is the $x(z)$ component of the force (torque) per unit area acting on the plane perpendicular to the $y$ axis.

The coefficient $\mu$ in Eq. (7) corresponds to the kinetic viscosity in dilute gas, which we can find an estimate of kinetic theory in textbooks on statistical physics, e.g. Ref. [7]. It is given by

$$
\mu \sim n \bar{v} m l \sim \frac{1}{\sigma} \sqrt{T m},
$$

where $l$ is the mean free path and $\bar{v}$ is the mean square displacement of the velocity in the $y$ direction. Here, $T$ is the granular temperature and the relation $l \sim 1 /(n \sigma)$ in two-dimensional system is used.

The coefficient $\mu_{B}$ is estimated by a similar argument to that of $\mu$. Because $C_{y z}$ represents the net angular momentum transfer per unit time per unit length due to the microrotation by particles crossing the plane $y=$ const, we can use the argument for $\mu$ by replacing $u(y)$ and $m$ by $\omega(y)$ and $I$, respectively. Then $\mu_{B}$ is estimated as

$$
\mu_{B} \sim n \bar{v} I l \sim \sigma \sqrt{T m},
$$

with $I=m \sigma^{2} / 8$ for a two-dimensional disk. As we have mentioned, the dimensions of $\mu$ and $\mu_{B}$ are different.

For an estimation of $\mu_{r}$, which gives coupling between particles' own spin and velocity field, we consider the collision of two disks near the plane $y=$ const [8]. If the surface of the disk has some roughness, the momentum tangent to the relative position of the colliding particles at the time of contact is transferred from one particle to another. It is plausible to assume that the tangential momentum transferred in one collision is proportional to $m$ times $\Delta u$, the relative tangential velocity of each particle at the contact point. In order to simplify the estimation, let us consider the situation with a uniform velocity field, namely $u^{\prime}(y)=0$ [9]. Then $\Delta u$ is given by $\Delta u \sim 2(\sigma / 2) \omega(y)$. Because the frequency of collision per unit time per unit length near the plane $y=$ const with the width $\sigma$ is proportional to $n^{2} \bar{v} \sigma^{2}$ in two-dimensions, the momentum transfer across the plane by collisions is estimated as

$$
\Delta M \sim n^{2} \bar{v} \sigma^{3} m[2 \omega(y)] .
$$

Comparing Eq. (11) and the second term of Eq. (7) with $u^{\prime}(y)=0$, we obtain

$$
\mu_{r} \sim n^{2} \bar{v} \sigma^{3} m \sim n^{2} \sigma^{3} \sqrt{T m} .
$$

Summarizing the results above, which are consistent with Ref. [0], we get following expressions for the coefficients of viscosity;

$$
\frac{\mu_{B}}{\mu_{r}} \sim \frac{1}{n^{2} \sigma^{2}} \sim l^{2}, \quad \frac{\mu}{\mu_{r}} \sim \frac{1}{\left(n \sigma^{2}\right)^{2}} \sim\left(\frac{l}{\sigma}\right)^{2} .
$$

It should be noted that, because the dimension of $\mu_{B}$ is different from that of $\mu$ and $\mu_{r}$, we need to introduce another length scale to characterize the macroscopic flow behavior in order to compare them. On the other hand, we can see that, when the number density is high enough, $\mu_{r}$ becomes comparable to $\mu$, then the coupling between the angular momentum and the linear momentum should play an important role.

Now we present the uniform, steady solution of the micropolar fluid equations (1), (2), and (3) on a slope, and compare the obtained profiles with the result of numerical simulation.

Let us consider the two-dimensional steady flow on a slope under the gravity. We take the $x$ axis in the direction down the slope, and the $y$ axis in the direction perpendicular to the slope. The inclination angle of the slope is $\theta$, and the acceleration of gravity $\boldsymbol{g}=(g \sin \theta,-g \cos \theta, 0)$. In the uniform, steady flow, $m n=\rho=\rho(y), \boldsymbol{v}=(u(y), 0,0)$, and $\boldsymbol{\omega}=(0,0, \omega(y))$, therefore the equation of continuity (11) is automatically satisfied. Eqs. (2) and (3) reduce to following differential equations:

$$
\begin{array}{r}
\rho g \sin \theta+\frac{\mathrm{d}}{\mathrm{d} y}\left[\mu \frac{\mathrm{d} u}{\mathrm{~d} y}+\mu_{r}\left(\frac{\mathrm{d} u}{\mathrm{~d} y}+2 \omega\right)\right]=0, \\
-\rho g \cos \theta-\frac{\mathrm{d} p}{\mathrm{~d} y}=0,
\end{array}
$$



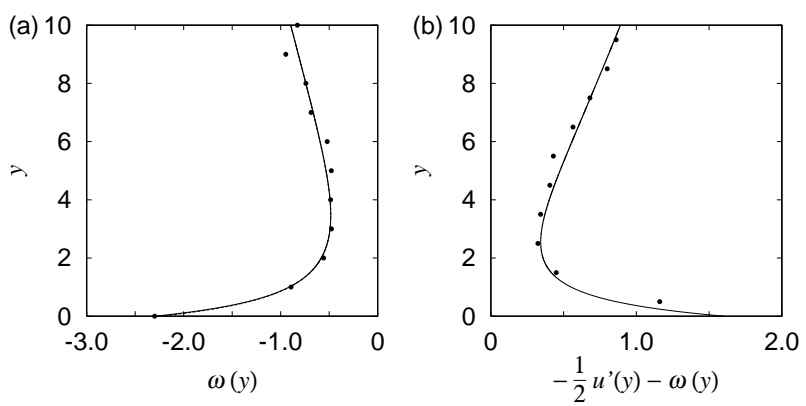

FIG. 1: The properties of the uniform flow. Filled circles show the data from the simulation and the solid line shows the uniform, steady solution of the micropolar fluid equations. (a) The angular velocity profile $\omega(y)$. The dashed line is the finite series approximation of the solution Eq. (22) with $k=1$ to 5 ; the difference from the solid line is hardly distinguished by the eye. (b) The profile of the deviation of the microrotation from the rotation of velocity field, $\frac{1}{2} \nabla \times \boldsymbol{v}-\boldsymbol{\omega}$.

and

$$
-2 \mu_{r}\left(\frac{\mathrm{d} u}{\mathrm{~d} y}+2 \omega\right)+\frac{\mathrm{d}}{\mathrm{d} y}\left[\mu_{B} \frac{\mathrm{d} \omega}{\mathrm{d} y}\right]=0
$$

The equation of state for a two-dimensional granular gas has been derived in Ref. [10], but here we adopt the lowest order estimate, namely $p=\rho T / m$. With the aid of the assumption of constant temperature $T=\bar{T}$, we obtain the density profile from Eq. (15);

$$
\rho=\rho_{0} \exp \left(-\frac{y}{h}\right), h=\frac{\bar{T}}{m g \cos \theta} .
$$

Then, from the estimate of the coefficients Eq. 113), we introduce the non-dimensional constants $\alpha$ and $\beta$ as

$$
\frac{\mu_{r}}{\mu}=\alpha \exp (-2 y / h), \quad \frac{\mu_{B}}{\mu}=\beta \sigma^{2} .
$$

After integrating Eqs. (14) and (16) with Eqs. (17) and (18), we obtain the following relation between the velocity field $u(y)$ and the microrotation field $\omega(y)$;

$$
\tilde{u}(Y)=-\exp (-Y)-\frac{\beta \epsilon^{2}}{2} \frac{\mathrm{d} \tilde{\omega}(Y)}{\mathrm{d} Y}+A_{0}
$$

where

$$
\tilde{u}=\frac{u}{\rho_{0} g h^{2} \sin \theta / \mu}, \quad \tilde{\omega}=\frac{\omega}{\rho_{0} g h \sin \theta / \mu},
$$

with $Y=y / h$ and $\epsilon=\sigma / h$. Here, $A_{0}$ is an integration constant. In the integration of Eq. (14), we imposed the boundary condition for stress tensor at the free surface, $\lim _{y \rightarrow \infty} S_{y x} \rightarrow 0$. From Eqs. (16) and (19), we have

$$
\frac{\mathrm{d}^{2} \tilde{\omega}(Y)}{\mathrm{d} Y^{2}}-2 \frac{\alpha \exp (-2 Y)}{\beta \epsilon^{2}}\left[\frac{\exp (-Y)+2 \tilde{\omega}(Y)}{1+\alpha \exp (-2 Y)}\right]=0 .
$$

Its general solution is given as the sum of a particular solution $\tilde{\omega}_{p}$ and two homogeneous solutions $\tilde{\omega}_{1}$ and $\tilde{\omega}_{2}$ by

$$
\tilde{\omega}(Y)=A \tilde{\omega}_{1}(Y)+B \tilde{\omega}_{2}(Y)+\tilde{\omega}_{p}(Y),
$$

with integration constants $A$ and $B$. Changing the variable from $Y$ to $\eta=\exp (-Y)$, we can obtain the expressions

$$
\begin{aligned}
\tilde{\omega}_{p} & =\sum_{k=1}^{\infty} a_{2 k+1} \eta^{2 k+1}, \quad \text { with } \quad a_{3}=\frac{2 \alpha}{9 \beta \epsilon^{2}}, \\
a_{2 k+1} & =f_{2 k+1} a_{2 k-1}, \\
\tilde{\omega}_{1} & =\sum_{k=0}^{\infty} b_{2 k} \eta^{2 k}, \quad \text { with } \quad b_{0}=1, \\
b_{2 k} & =f_{2 k} b_{2 k-2},
\end{aligned}
$$

and

$$
\begin{aligned}
& \tilde{\omega}_{2}=\tilde{\omega}_{1} \log \eta+\sum_{k=1}^{\infty} c_{2 k} \eta^{2 k}, \quad \text { with } \quad c_{0}=0, \\
& c_{2 k}=f_{2 k} c_{2 k-2}-\frac{\alpha}{k^{3}}\left[\frac{1}{\beta \epsilon^{2}}+k-1\right] b_{2 k-2},
\end{aligned}
$$

where

$$
f_{m} \equiv \frac{\alpha}{m^{2}}\left[\frac{4}{\beta \epsilon^{2}}-(m-2)^{2}\right] .
$$

Note that $\tilde{\omega}_{p}$ and $\tilde{\omega}_{1}$ are regular but $\tilde{\omega}_{2}$ diverges at $\eta=0$ $(Y \rightarrow \infty)$.

Now we show that the solution obtained from the micropolar fluid model can quantitatively reproduce the results of numerical simulations of collisional granular flow on a bumpy slope [11. In the simulation, the discrete element method is employed with normal and tangential elastic force and dissipation. The particles are modeled by disks with diameter $\sigma$, and the parameters are chosen so that the normal restitution $e_{n}$ becomes 0.7 . The surface of the slope is made rough by attaching identical particles to it. In the following, all quantities are given in dimensionless form with mass unit $m$, length unit $\sigma$, and time unit $\tau=\sqrt{\sigma / g}$.

We used the data of the simulation with inclination $\sin \theta=0.45$, system size $L=1002$ and number of flowing particle $N=1000$. In the simulations, uniform flow is realized during $500 \lesssim t \lesssim 2000$ where $t$ is the time [11]. In order to determine the profiles of the mean quantities describing the flow, we divide the space into layers which are one particle diameter wide parallel to the slope, calculate the averages inside layers, and then average the data over the time within the uniform flow, $1000 \leq t<1500$. The origin $y=0$ is taken one diameter above from the top of the disks attached to the slope.

Now let us compare our analytical result from the micropolar fluid model with the simulation data. First, we 
confirmed that the number density profile can be wellfitted by Eq. (17) with $h=2.24$, namely $\bar{T} \sim 2.0$, which has been checked to be close to the averaged value of $T$. In order to fit the solutions (19) and (22) to the simulation data, we determine the boundary values $u(0)$ and $\omega(0)$ from the data and treat $\alpha, \beta, \mu / \rho_{0}$ and $\omega^{\prime}(0)$ as fitting parameters. From Fig. 1 (a), we can see that the micropolar fluid equations reproduce the angular velocity quantitatively. Here, the value of parameters are $\alpha \sim 0.10, \beta \sim 0.12, \mu / \rho_{0} \sim 0.95$, and $\omega^{\prime}(0) \sim 2.9$. The velocity profile $u(y)$ can also be well-reproduced by the solution (19); however, because the density is low, the deviation of the velocity profile from the solution of the Navier-Stokes equation, i.e. $\tilde{u}(Y)=-\exp (-Y)+A_{0}$, is small. Actually, we have checked that the data can be reasonably fit with any small value of $\alpha$, as long as $\alpha / \beta$ is chosen appropriately. However, it is significant that the solution (22) can reproduce the sharp variation of $\omega(y)$ over only a few diameters near the base.

The more important thing is that the mean spin $\boldsymbol{\omega}$ deviates systematically from the rate of bulk rotation $\frac{1}{2} \nabla \times \boldsymbol{v}$, and that the micropolar fluid model can reproduce this deviation. In Fig. 目 (b), we see that the deviation is large near the base, because each particle is forced to rotate by the collision with the slope. This result indicates the importance of the couple stress near the boundary, as pointed out in Ref. [12]. This deviation may produce the velocity profile different from the Newtonian fluids described by the Navier-Stokes equation due to the coupling between the spin of each particle and the linear velocity field [13]. On the other hand, it seems the deviation also becomes large in the region far from the boundary. The reason is that the microrotation field in this region is dominated by a small number of particles spins that are generated by collisions with the boundary.

In summary, the micropolar fluid model has been applied to a collisional granular flow with relatively low density. It has been demonstrated that the solution for uniform, steady flow on an inclined surface reproduces the results of numerical simulation.

Because the density is low, the ideal gas assumption for the equation of state and the estimation of the viscosity from the elementary kinetic theory work well. In order to apply the model to denser collisional flows, we need a systematic extension of the theory using, for example, Enskog theory. This has already been done in the context of polyatomic fluids for completely rough spheres without energy dissipation [5] and it should be possible to extend such results to the dissipative case [6]. The equation of state for dense granular gas has also been discussed recently [14]. On the other hand, it is known that the effect of the particle spin becomes more important in dense frictional flows [15]. Much research has been done to construct the mechanics of granular media which is valid not only for collisional flow but also for denser situations based on the micropolar or Cosserat theory [16]. The concepts of micropolar mechanics may help to link the understandings of the collisional and the frictional flows of granular materials.

The authors are grateful to J. T. Jenkins for a careful reading of the manuscript. N. M. and $\mathrm{H}$. H. also thank him and A. Shimosaka for fruitful discussions. N. M. and H. N. are grateful for the warm hospitality during their stay at YITP of Kyoto University where part of this work has been carried out.

[1] L. P. Kadanoff, Rev. Mod. Phys. 71, 435 (1999).

[2] J. T. Jenkins and S. B. Savage, J. Fluid Mech. 130, 187 (1983); C. S. Campbell, Ann. Rev. Fluid Mech. 22, 57 (1990); I. Goldhirsch, in Modeling in Applied Sciences: A Kinetic Theory Approach, ed. N. Bellomo and M. Pulvirenti (Birkhäuser, Boston, 2000), and references therein.

[3] D. W. Condiff and J. S. Dahler, Phys. Fluids 7, 842 (1964); A. C. Eringen, J. Math. Mech. 16, 1 (1966); G. Lukaszewicz, Micropolar Fluids: Theory and Applications (Birkhäuser, Boston, 1999); H. Hayakawa, Phys. Rev. E 61, 5477 (2000), and references therein.

[4] K. Kanatani, Trans. Jpn. Soc. Mech. Eng. B 45, 507, 515 (1979); J. Kano, A. Shimosaka, and J. Hidaka, J. Soc. Powder Techol. Jpn. 33, 95 (1996).

[5] B. J. McCoy, S. I. Sandler, and J. S. Dahler, J. Chem. Phys. 453485 (1966); J. S. Dahler and M. Theodosopulu, Adv. Chem. Phys. 31, 155 (1975).

[6] C. K. K. Lun, J. Fluid Mech. 233, 539 (1991).

[7] F. Reif, Berkeley physics course vol.5, Statistical Physics, (McGraw-Hill, New York, 1965).

[8] It should be noted that, such a collision process produces the (angular) momentum transfer related to not only $\mu_{r}$ but also $\mu\left(\mu_{B}\right)$. In this paper, only the lowest order in each viscosity is considered, therefore such corrections to $\mu$ and $\mu_{B}$ are neglected.

[9] If we consider the situation with $u^{\prime}(y) \neq 0$, we get $\Delta u=$ $\sigma\left[u^{\prime}(y) \cos ^{2} \theta+\omega(y)\right]$, where $\theta$ is the angle between the $y$ axis and the vector along the centerline from one particle to another. We need careful calculation in the average process of $\theta$ which is beyond elementary kinetic theory. We confirmed that a more systematic derivation of $\mu_{r}$ similar to the method in Ref. [6] gives the asymmetric part of the stress tensor proportional to $u^{\prime}(y)+2 \omega$.

[10] J. T. Jenkins and M. W. Richman, Phys. Fluids 28, 3485 (1985).

[11] N. Mitarai and H. Nakanishi, J. Phys. Soc. Jpn. 70, 2809 (2001).

[12] C. S. Campbell, J. Fluid. Mech. 247, 111 (1993).

[13] J. T. Jenkins, J. Appl. Mech. 59, 120 (1992).

[14] S. Luding, Phys. Rev. E 63, 042201 (2001).

[15] C. T. Veje, D. W. Howell, and R. P. Behringer, Phys. Rev. E 59, 739 (1999); D. M. Mueth et al., Nature 406, 385 (2000); M. Lätzel, S. Luding, and H. J. Herrmann, Gran. Matt. 2, 123 (2000).

[16] J. P. Bardet and I. Vardoulakis, Int. J. Solids Struct., 38, 353 (2001); H. B. Mühlhaus and P. Hornby, Tectonophys. 335, 63 (2001), and references therein. 